\title{
Numerical Simulation and Optimization of Perforated Tube Trolley in Circular Cooler
}

\author{
Bin Wu, Kunrong Huang*, Defan Qing, Maokui Zhu \\ School of Mechanical Engineering, University of South China, Hengyang, China \\ Email: *624673031@qq.com
}

How to cite this paper: $\mathrm{Wu}, \mathrm{B}$., Huang, K.R., Qing, D.F. and Zhu, M.K. (2017) Numerical Simulation and Optimization of Perforated Tube Trolley in Circular Cooler. World Journal of Engineering and Technology, 5, 684-695.

https://doi.org/10.4236/wjet.2017.54057

Received: September 16, 2017

Accepted: November 7, 2017

Published: November 10, 2017

Copyright (c) 2017 by authors and Scientific Research Publishing Inc. This work is licensed under the Creative Commons Attribution International License (CC BY 4.0).

http://creativecommons.org/licenses/by/4.0/

\begin{abstract}
Three symmetrically perforated tubes were arranged in the circular cooler trolley as auxiliary cooling inlet to improve the cooling performance of the sintered body during the production process. Fluent 15.0 has been used to simulate the process; the study shows that the perforated tube structure trolley has changed the temperature field within the sintering area, thereby improving the sintering area of the cooling effect and uniformity, also greatly reducing the cooling time. Compared with the traditional trolley, the best structure of the porous tube trolley has reduced $41 \%$ cooling time and increased $50 \%$ waste heat recovery.
\end{abstract}

\section{Keywords}

Circular Cooler, Perforated Tube, Structural Optimization, Cooling Performance, Orthogonal Test

\section{Introduction}

In the iron and steel production process, the circular cooler is the most widely used equipment for the sintering process [1]. The sintering furnace cooling process directly determines the development of iron and steel enterprises. After decades of development and research, the equipment manufacturing process has become more and more matured [2]. FENG Junxiao has established twodimensional heat transfer model of sintered porous media. After simulation, he got the best operating conditions of the parameters, the thickness of the material layer and the particle size [3]. The parameters such as the thickness and air volume of the sintering process were studied by Luo Yuanqiu. The results showed that the air volume and the thickness have an optimal value [4]. The gas-solid heat transfer coefficient of sintered ore in different working conditions was stu- 
died experimentally by ZHAO Bin. The porosity and the Nusselt number were sorted according to the experimental data. Through the ring cooling machine sintered ore channel gas-solid heat test, he has got the relationship between the product and the Reynolds number Curve [5]. The three-dimensional physical model of the two-energy equation of local non-thermodynamically balanced heat transfer is established by ZHANG Xiaohui. The numerical simulation of the two-phase heat transfer process and its influence parameters is carried out by using Fluent simulation software. And the orthogonal test method was used to combine the optimal parameters, and finally the conclusion of the experiment was used [6].

However, the cooling process of the circular cooler trolley which layer by layer is still relatively simple, resulting in poor effect of the top area of the sinter and the longer cooling time. This paper starts from the internal structure of the trolley to make the design and optimization of the structure so that the cooling time of the sinter is greatly reduced and the recovery of waste heat is greatly improved.

\section{Research Model}

$450 \mathrm{~m}^{2}$ sintering ring cooling machine as the research object, the size of the trolley $4 \mathrm{~m}, 2.5 \mathrm{~m}, 1.6 \mathrm{~m}$. During the working process, the circular cooler trolley can be regarded as fixed, because the running speed of the tank is much lower than that of the air passing through the trolley. This process can be treated as a fixed bed of gas-solid heat exchange in the numerical simulation [7]. The built-in perforated tube trolley consists of a trolley wall, a grate plate and three perforated tubes. Below the grate plate, there are the main inlet cooling inlet, and the three sides of the three tubes are used as auxiliary cooling inlet. In order to facilitate the simulation calculation, the three-dimensional model of the trolley is simplified as a two-dimensional model. Built-in perforated tube trolley structure diagram and vertical sectional view as Figure 1 and Figure 2 shown.

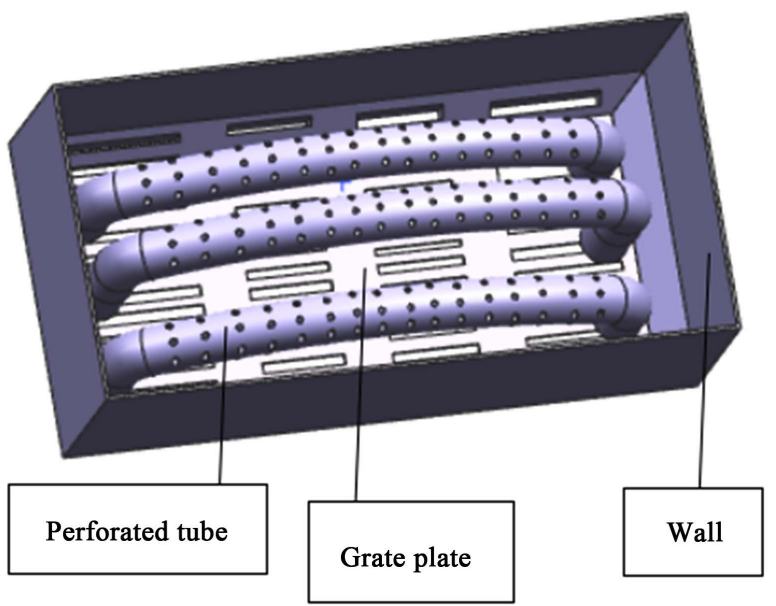

Figure 1. Schematic diagram of the built-in perforated tube structure of the circular cooler. 


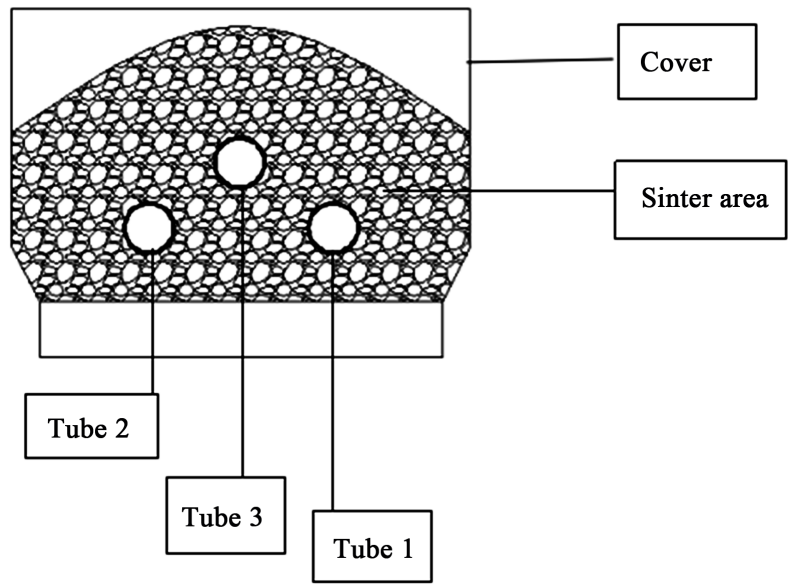

Figure 2. Vertical sectional view of the ring trolley structure model.

\section{Theoretical Basis and Basic Assumptions}

In the process of cooling, there are heat conduction, convection and radiation. [8] Therefore, the heat exchange in the circular cooler can be solved by the equations of continuity equation, momentum conservation equation and energy conservation equation to describe.

\subsection{Continuity Equation}

According to the law of conservation of mass, the continuity equation can be obtained:

$$
\frac{\partial \rho}{\partial t}+\frac{\partial(\rho u)}{\partial x}+\frac{\partial(\rho v)}{\partial y}+\frac{\partial(\rho w)}{\partial z}=0
$$

In the above equation, $\rho$ is the density; $t$ is the time; $u, v$ and $w$ is the component of the velocity vector, respectively $x, y$ and $z$ in the direction.

\subsection{Momentum Conservation Equation}

$$
\begin{aligned}
& \frac{\partial(\rho u)}{\partial t}+\frac{\partial(\rho u u)}{\partial x}+\frac{\partial(\rho u v)}{\partial y}+\frac{\partial(\rho u w)}{\partial z} \\
& =\frac{\partial}{\partial x}\left(\mu \frac{\partial u}{\partial x}\right)+\frac{\partial}{\partial y}\left(\mu \frac{\partial u}{\partial y}\right)+\frac{\partial}{\partial z}\left(\mu \frac{\partial u}{\partial z}\right)-\frac{\partial p}{\partial x}+S_{u} \\
& \frac{\partial(\rho v)}{\partial t}+\frac{\partial(\rho v u)}{\partial x}+\frac{\partial(\rho v v)}{\partial y}+\frac{\partial(\rho v w)}{\partial z} \\
& =\frac{\partial}{\partial x}\left(\mu \frac{\partial v}{\partial x}\right)+\frac{\partial}{\partial y}\left(\mu \frac{\partial v}{\partial y}\right)+\frac{\partial}{\partial z}\left(\mu \frac{\partial v}{\partial z}\right)-\frac{\partial p}{\partial y}+S_{v} \\
& \frac{\partial(\rho w)}{\partial t}+\frac{\partial(\rho w u)}{\partial x}+\frac{\partial(\rho w v)}{\partial y}+\frac{\partial(\rho w w)}{\partial z} \\
& =\frac{\partial}{\partial x}\left(\mu \frac{\partial w}{\partial x}\right)+\frac{\partial}{\partial y}\left(\mu \frac{\partial w}{\partial y}\right)+\frac{\partial}{\partial z}\left(\mu \frac{\partial w}{\partial z}\right)-\frac{\partial p}{\partial z}+S_{w}
\end{aligned}
$$

In the above equation, $\rho$ is the density; $t$ is the time; $u, v$ and $w$ is 
the velocity vector in the $x, y$ and $z$ direction of the component; $p$ is the pressure on the fluid micro-body; $\mu$ is the dynamic viscosity; $S_{u}, S_{v}$ and $S_{w}$ is the momentum conservation equation of the generalized source.

\subsection{Energy Conservation Equation}

Numerical simulations for built-in porous trolleys can be considered as porous media models. Without considering convection acceleration terms and diffusion terms, according to Darcy's law [9], the energy equation for porous media is as follows:

$$
\begin{aligned}
& \frac{\partial}{\partial t}\left[\varepsilon \rho_{f} E_{f}+(1-\varepsilon) \rho_{s} E_{s}\right]+\nabla\left[\boldsymbol{u} \cdot\left(\rho_{f} E_{f}+p\right)\right] \\
& =S_{f}^{h}+\nabla\left[k_{\text {eff }} \nabla T-\left(\sum_{i} h_{i} J_{i}\right)+(\overline{\bar{\tau}} \cdot \boldsymbol{u})\right]
\end{aligned}
$$

$E_{f}$ is the total energy of the fluid; $\rho_{f}$ is the density of the fluid; $E_{s}$ is the total energy of the solid medium; $\rho_{s}$ is the density of the solid medium; $\varepsilon$ is the porosity; $T$ is the temperature; $k_{\text {eff }}$ is the effective thermal conductivity of the porous medium; $S_{f}^{h}$ is the source of the enthalpy of the fluid.

\subsection{Basic Assumptions}

Because the circulation, heat transfer and mass transfer process are very complicated [10], in order to facilitate the numerical simulation of the sinter in the trolley, the following assumptions are put forward:

1) The sinter particles are idealized particles and are evenly distributed in the trolley, there is no temperature difference between the particles;

2) The wall in the trolley does not participate in heat transfer;

3) The operation parameters of the cooling trolley are not changed with time.

After making the assumptions, the traditional trolley is simulated. The results are compared with the relevant experimental papers, the results found that the error within $10 \%$, so that the assumption is valid.

\section{Optimization Design and Performance Analysis}

\subsection{Optimized Design}

Put forward four factors: 1) Three pipe diameter $d ; 2$ ) both sides of the tube from the grate plate height $\left.h_{1} ; 3\right)$ the middle tube from the grate plate height $\left.h_{2} ; 4\right)$ the distance between the two sides of the tube from the side $l$. Those factors constitute a four factors and three levels orthogonal experiment. Combined with the trolley structure and perforated tube for the seamless steel pipe and other factors, perforated tube diameter ranges $200 \mathrm{~mm}$ from $400 \mathrm{~mm}$, set $d$ $=270 \mathrm{~mm}, 310 \mathrm{~mm}, 350 \mathrm{~mm}$; taking the sinter stacking height and center layout principle into account, $h_{1} 250-500 \mathrm{~mm}$ and $h_{2} 550-950 \mathrm{~mm}$ for the best, take $h_{1}$ were $320 \mathrm{~mm}, 370 \mathrm{~mm}, 420 \mathrm{~mm}, h_{2}$ were $640 \mathrm{~mm}, 740 \mathrm{~mm}, 840 \mathrm{~mm}$; taking into account the sinter no clogging problems, $l$ should range $400 \mathrm{~mm}$ 
from $800 \mathrm{~mm}$, take $500 \mathrm{~mm}, 600 \mathrm{~mm}, 700 \mathrm{~mm}$. Structure parameters of calculation area shown in Figure 3.

The inlet air velocity is $1.1 \mathrm{~m} / \mathrm{s}$, the inlet temperature is $298 \mathrm{~K}$, the initial temperature of sinter is $1073 \mathrm{~K}$, and $373 \mathrm{~K}$ is set as cooling node. According to the principle of orthogonal test, select the nine groups of numerical combinations. Nine groups of perforated tube trolley were simulated by Fluent respectively. The value selection of the nine groups of study objects and the cooling time of the sinter from $1073 \mathrm{~K}$ to $373 \mathrm{~K}$ are shown in Table 1.

According to the Fluent simulation calculation result, the cooling time of the traditional trolley sinter from $1073 \mathrm{~K}$ to $373 \mathrm{~K}$ is $2130 \mathrm{~s}$. It can be seen that the trolley built-in three porous tube reduced the average cooling time by $45 \%$ compared with the traditional one.

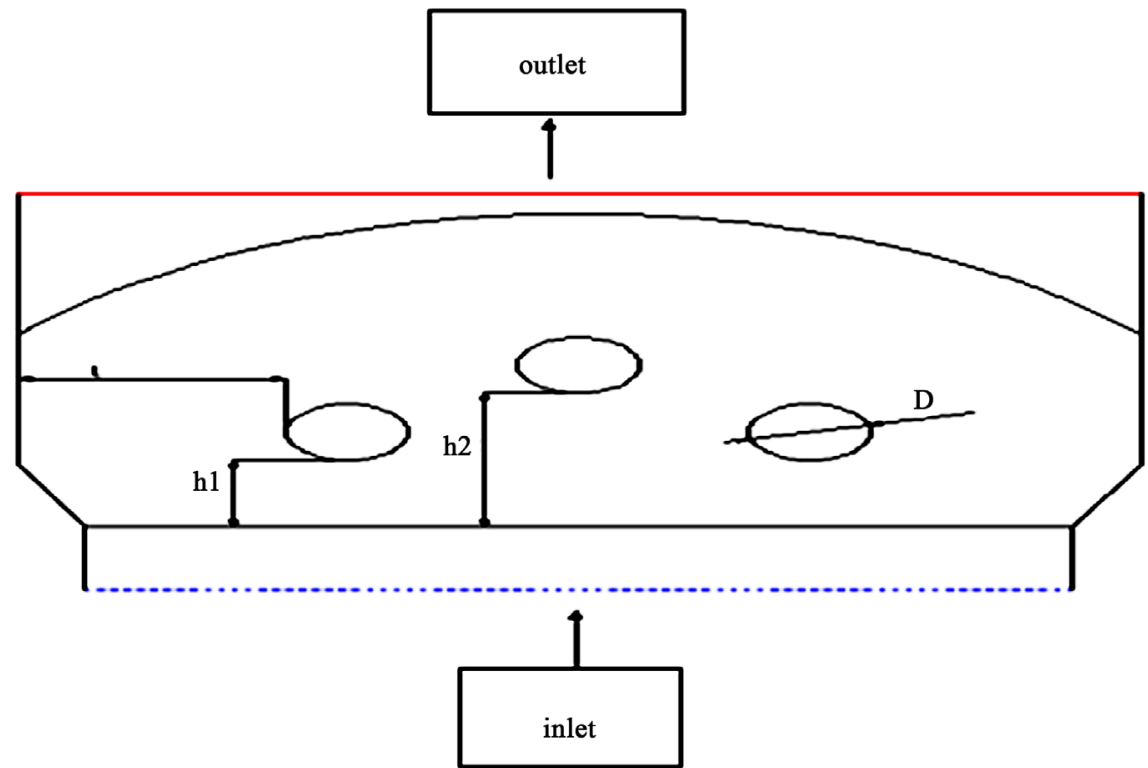

Figure 3. Structure parameters of calculation area.

Table 1. 9 sets of study object numerical parameters and cooling time.

\begin{tabular}{cccccc}
\hline Group No & $d(\mathrm{~mm})$ & $h_{1}(\mathrm{~mm})$ & $h_{2}(\mathrm{~mm})$ & $l(\mathrm{~mm})$ & Cooling time $(\mathrm{s})$ \\
\hline 1 & $1(270)$ & $1(320)$ & $1(640)$ & $1(500)$ & 1200 \\
2 & 1 & $2(370)$ & $2(740)$ & $2(600)$ & 1225 \\
3 & 1 & $3(420)$ & $3(840)$ & $3(700)$ & 1405 \\
4 & $2(310)$ & 1 & 2 & 3 & 1100 \\
5 & 2 & 2 & 3 & 1 & 1210 \\
6 & 2 & 3 & 1 & 2 & 1160 \\
7 & $3(350)$ & 1 & 3 & 2 & 1150 \\
8 & 3 & 2 & 1 & 3 & 990 \\
9 & 3 & 3 & 2 & 1 & 1250 \\
\hline
\end{tabular}




\subsection{Analysis of Waste Heat Recovery Utilization Amount}

During the sintering production, great amount of sinter cooled air temperature ranges from $423 \mathrm{~K}$ to $623 \mathrm{~K}$ has high recovery value [11]. According to the calculation formula of waste heat recovery [12]:

$$
Q=c_{p} m \Delta T=\left(c_{p} \rho \Delta T\right) \cdot\left(q_{v} \cdot t\right)
$$

In the formula, $Q$ is the heat capacity of the waste heat recovery, $(\mathrm{kJ}) ; c_{p}$ is the specific heat capacity of the air, $0.725 \mathrm{~J} /\left(\mathrm{kg} \cdot{ }^{\circ} \mathrm{C}\right) ; \rho$ is the density of the air, $1.29 \mathrm{~kg} / \mathrm{m}^{3} ; \Delta T$ is the temperature difference of the recovered value of the exhaust gas; $q_{v}$ is the volumetric flow rate of the inlet velocity, $4.4 \mathrm{~m}^{3} / \mathrm{s} ; t$ is waste heat recovery stage of the time, (s).

Table 1 in the nine sets of fixed value of the import wind speed numerical simulation calculation, get the corresponding waste heat utilization section as shown in Table 2.

The four factors and three levels orthogonal test is used to analyze the waste heat utilization as shown in Table 3.

The greater amount of waste heat recovery, indicating that the more heat can be used, and the relative energy consumption in the cooling process less. The greater the difference is, indicating the impact of this level on the results of the greater, the level has more important influence on the result. So the importance order of four parameters is $l>d>h_{1}>h_{2}$. The higher the $K$ value is, the better level is in the factor. Select the largest set of data from $K$ to get the preferred group $d(3) h_{1}(3) h_{2}(3) l(1)$.

The simulation of the preferred group structure trolley, get up to $373 \mathrm{~K}$ when the time is $1155 \mathrm{~s}$, the waste heat recovery and utilization time is $490 \mathrm{~s}$, and the waste heat recovery is $403.28 \mathrm{~kJ}$. In the nine groups of structures, the waste heat recovery of Group 9 is the highest. Group 9 and the Preferred group cooling time, waste heat recovery compared to Figure 4 and Figure 5.

Through the comparison between the Preferred group and the Group 9, the waste heat recovery ratio of the Group 9 structure trolley is much higher than that of the Preferred group, and the cooling time is very close.

In the cooling time, the fastest cooling rate of $373 \mathrm{~K}$ is the Group 8 trolley, but the Group 8 can use the waste heat recovery is only $57 \%$ to Group 9.

In the comprehensive comparison, the Group 9 is selected as the optimal structure combination.

Table 2. Perforated tube trolley and traditional trolley waste heat utilization.

\begin{tabular}{ccccccccccc}
\hline $\begin{array}{c}\text { Group } \\
\text { number }\end{array}$ & 1 & 2 & 3 & 4 & 5 & 6 & 7 & 8 & 9 & $\begin{array}{c}\text { traditional } \\
\text { trolley }\end{array}$ \\
\hline time (s) & 365 & 345 & 350 & 290 & 470 & 375 & 400 & 330 & 575 & 145 \\
$\begin{array}{c}\text { Waste heat } \\
\text { recovery (kJ) }\end{array}$ & 300.40 & 283.94 & 288.06 & 238.68 & 386.82 & 308.63 & 329.21 & 271.60 & 473.24 & 119.34 \\
\hline
\end{tabular}


Table 3. Analysis of orthogonal test results.

\begin{tabular}{|c|c|c|c|c|c|}
\hline \multirow{2}{*}{$\begin{array}{l}\text { Test group } \\
\text { number }\end{array}$} & \multicolumn{5}{|c|}{ Test results } \\
\hline & $d$ & $h_{1}$ & $h_{2}$ & 1 & Waste heat recovery $(\mathrm{kJ})$ \\
\hline 1 & $1(270)$ & $1(320)$ & $1(640)$ & $1(500)$ & 300.40 \\
\hline 2 & 1 & $2(370)$ & $2(740)$ & $2(600)$ & 283.94 \\
\hline 3 & 1 & $3(420)$ & $3(840)$ & $3(700)$ & 288.06 \\
\hline 4 & $2(310)$ & 1 & 2 & 3 & 238.68 \\
\hline 5 & 2 & 2 & 3 & 1 & 386.82 \\
\hline 6 & 2 & 3 & 1 & 2 & 308.63 \\
\hline 7 & $3(350)$ & 1 & 3 & 2 & 329.21 \\
\hline 8 & 3 & 2 & 1 & 3 & 271.60 \\
\hline 9 & 3 & 3 & 2 & 1 & 473.24 \\
\hline K1 & 872.40 & 868.29 & 880.63 & 1160.46 & \\
\hline K2 & 934.13 & 942.36 & 995.86 & 921.78 & \\
\hline K3 & 1074.05 & 1069.93 & 1004.09 & 798.34 & \\
\hline $\bar{K}_{1}$ & 290.80 & 289.43 & 293.54 & 386.82 & \\
\hline $\bar{K}_{2}$ & 311.38 & 314.12 & 331.95 & 307.26 & \\
\hline $\bar{K}_{3}$ & 358.02 & 356.64 & 334.70 & 266.11 & \\
\hline Range & 67.22 & 67.21 & 41.16 & 120.71 & \\
\hline Primary order & \multicolumn{5}{|c|}{$l>d>h_{1}>h_{2}$} \\
\hline Excellent level & \multicolumn{5}{|c|}{$d(3), h_{1}(3), h_{2}(3), l(1)$} \\
\hline Excellent combination & \multicolumn{5}{|c|}{$d(3), h_{1}(3), h_{2}(3), l(1)$} \\
\hline
\end{tabular}

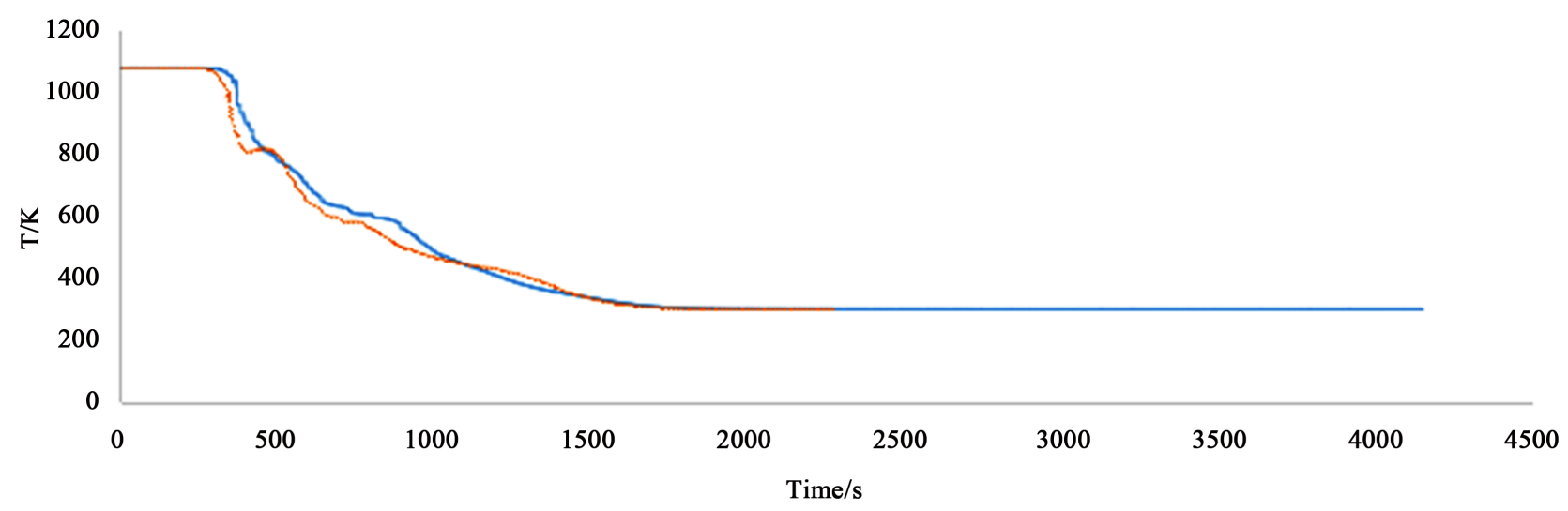

— Preferred group _............ Group 9

Figure 4. The cooling process of Group 9 and the preferred group. 


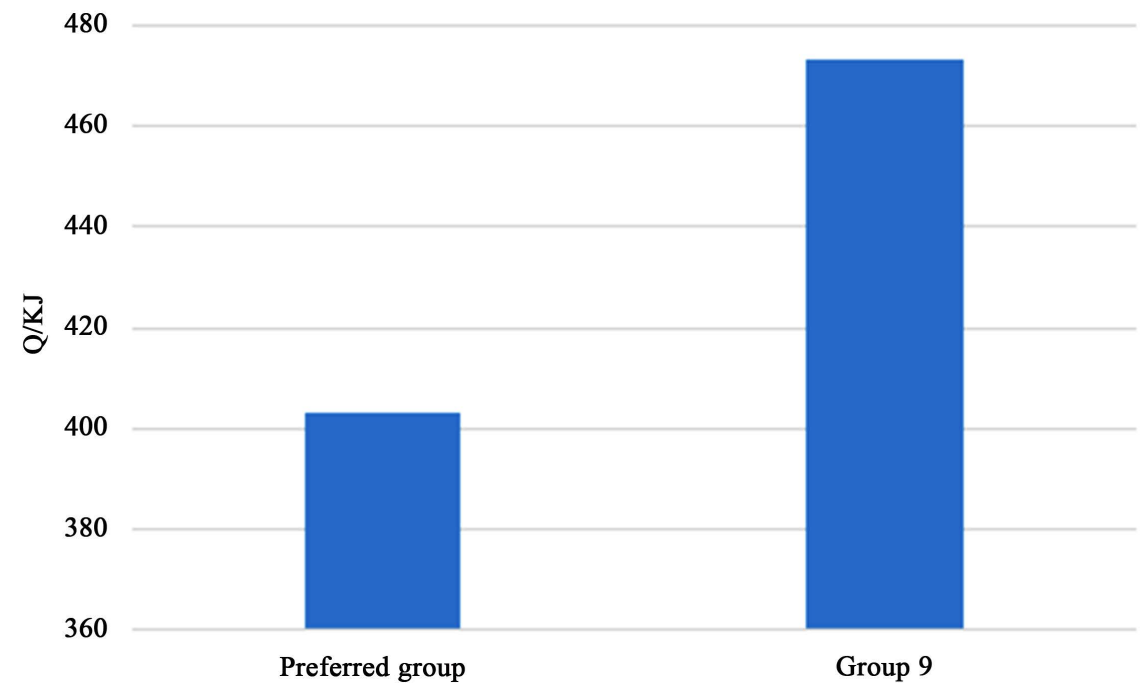

Figure 5. The waste heat recovery of group 9 and the preferred group.

\subsection{Comparison of Temperature Fields}

In order to obtain the real-time effect of the built-in perforated tube cooling process, the temperature field analysis were carried out for the Group 9 and the traditional group structure trolley. The cloud field of the Group 9 structure trolley and traditional structure trolley at 800 s, 1200 s and 1600 s are shown in Figures 6-11 by Fluent numerical simulation.

In the traditional structure of the trolley, the cooling air can only enter the sintered area from beneath the grate plate. During the process of sintering the area, the air is continuously heated, so the high temperature sinter is cooled from bottom to top. In the built-in perforated tube structure trolley, the cooling air is equivalent to four outlets-the grate plate and the full side of the perforated tube $1,2,3$. The upper of perforated tube part mainly by the perforated tube side of the cooling air to cool, the lower mainly from the grate plate into the cooling air to cool. Through the comparison of the temperature field of the Group 9 and the traditional trolley, it is found that the Group 9 trolley is more well-distributed than the traditional structure trolley in the sintering of the cooling.

\section{Conclusions}

1) Perforated tube structure trolley has greatly changed the temperature field of the cooling air in the sinter area, and accelerated the air flow velocity at the wall surface and the tapered area. It achieved the cooling effect better than that of the traditional structure trolley. It is of great significance to improve the cooling efficiency of the sinter and reduce the energy consumption during cooling.

2) The orthogonal test design method was used to construct the four factors and three levels test; the results show that the order of influence on the heat recovery is $l>d>h_{1}>h_{2}$. The optimal combination of three kinds of perforated tube trolley is the 9th group; the structural parameters are $d=350 \mathrm{~mm}, l=500$ $\mathrm{mm}, h_{1}=420 \mathrm{~mm}, h_{2}=740 \mathrm{~mm}$; Compared with the traditional trolley, the best 


\section{Temperature}

Contour 1

ANSYS

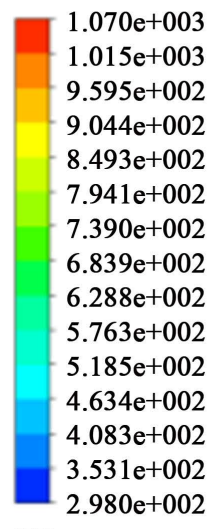

[K]
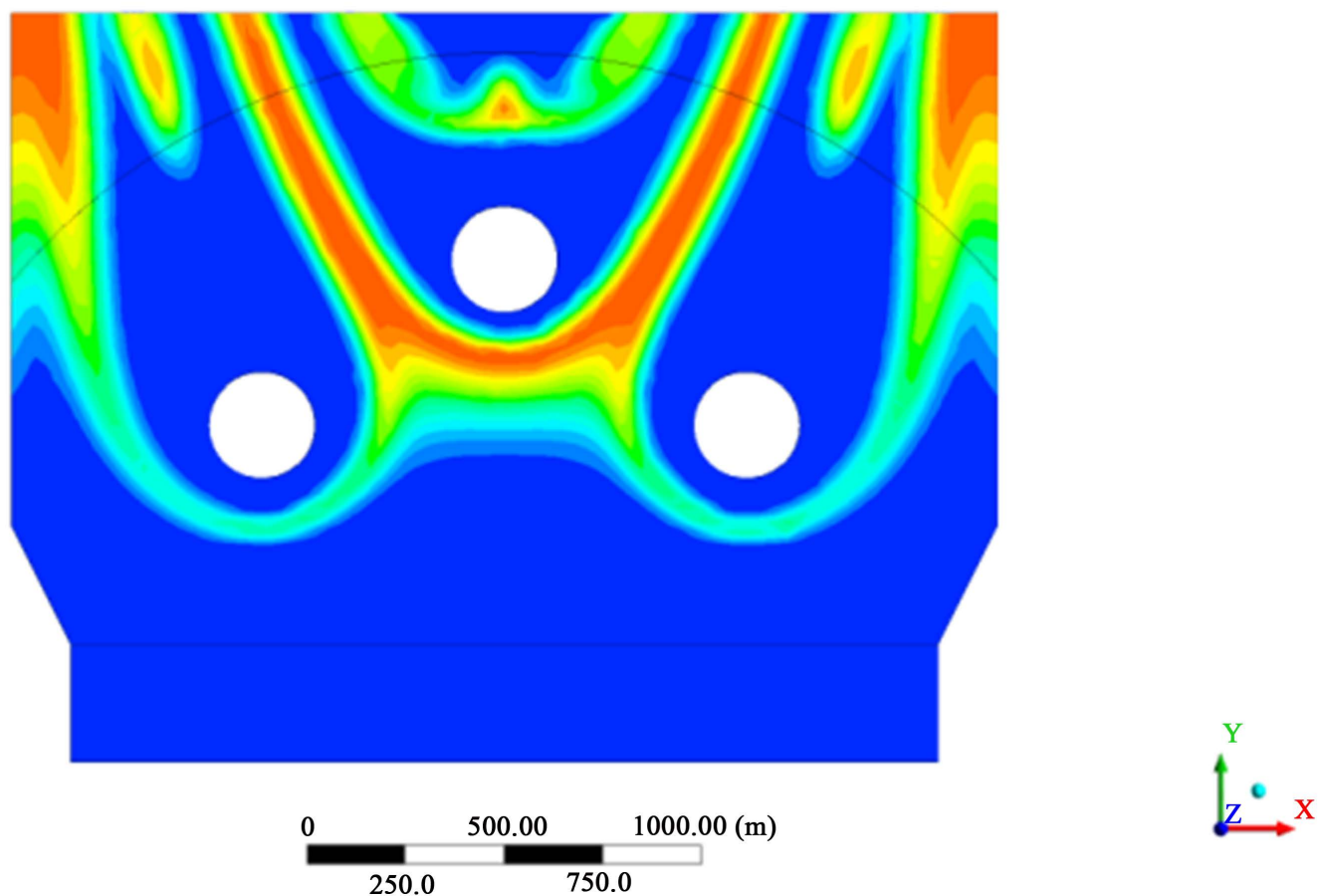

Figure 6. Group 9 structure trolley $800 \mathrm{~s}$ air temperature distribution cloud.

\section{Temperature}

Contour 2

ANSYS

R15.0

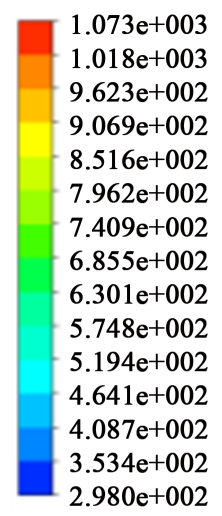

[K]

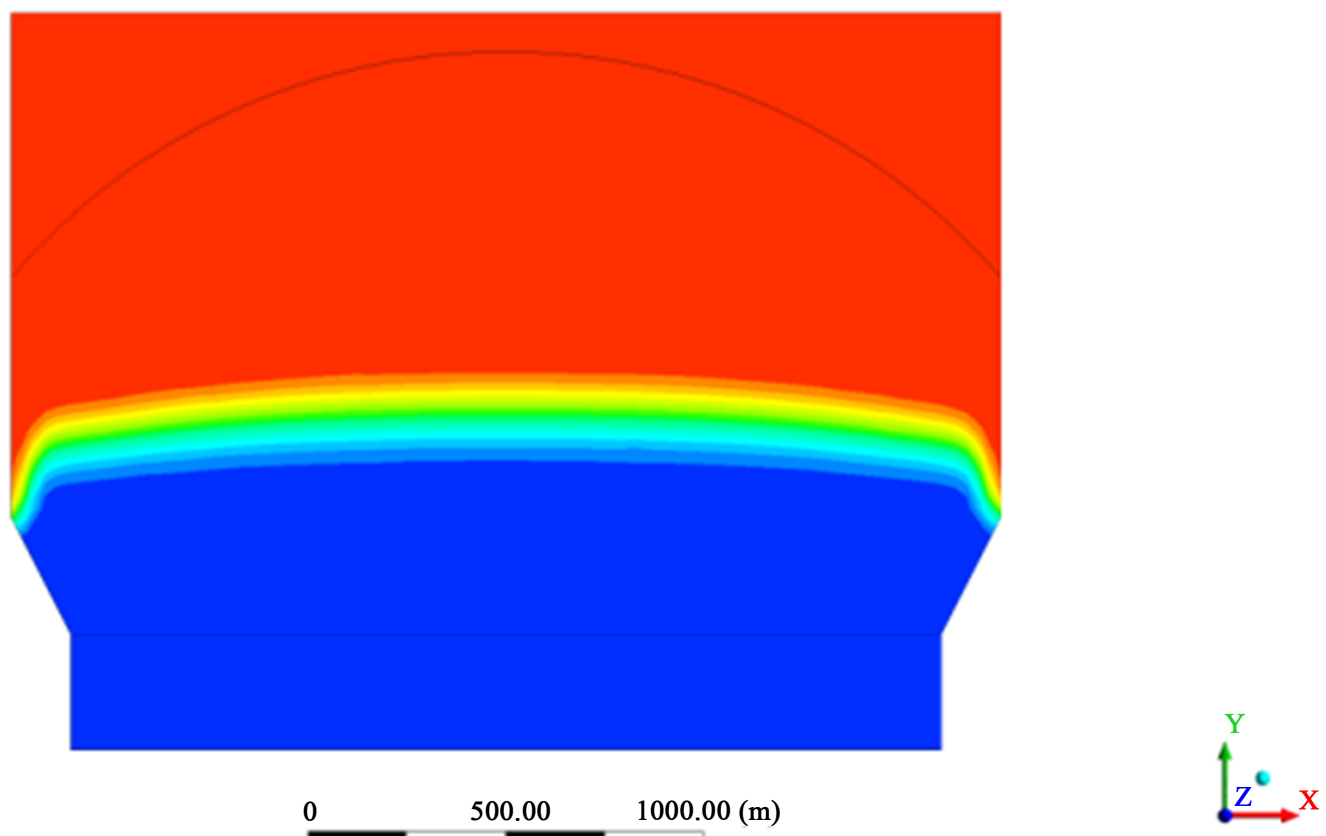

Figure 7. Traditional structure trolley $800 \mathrm{~s}$ air temperature distribution cloud. 

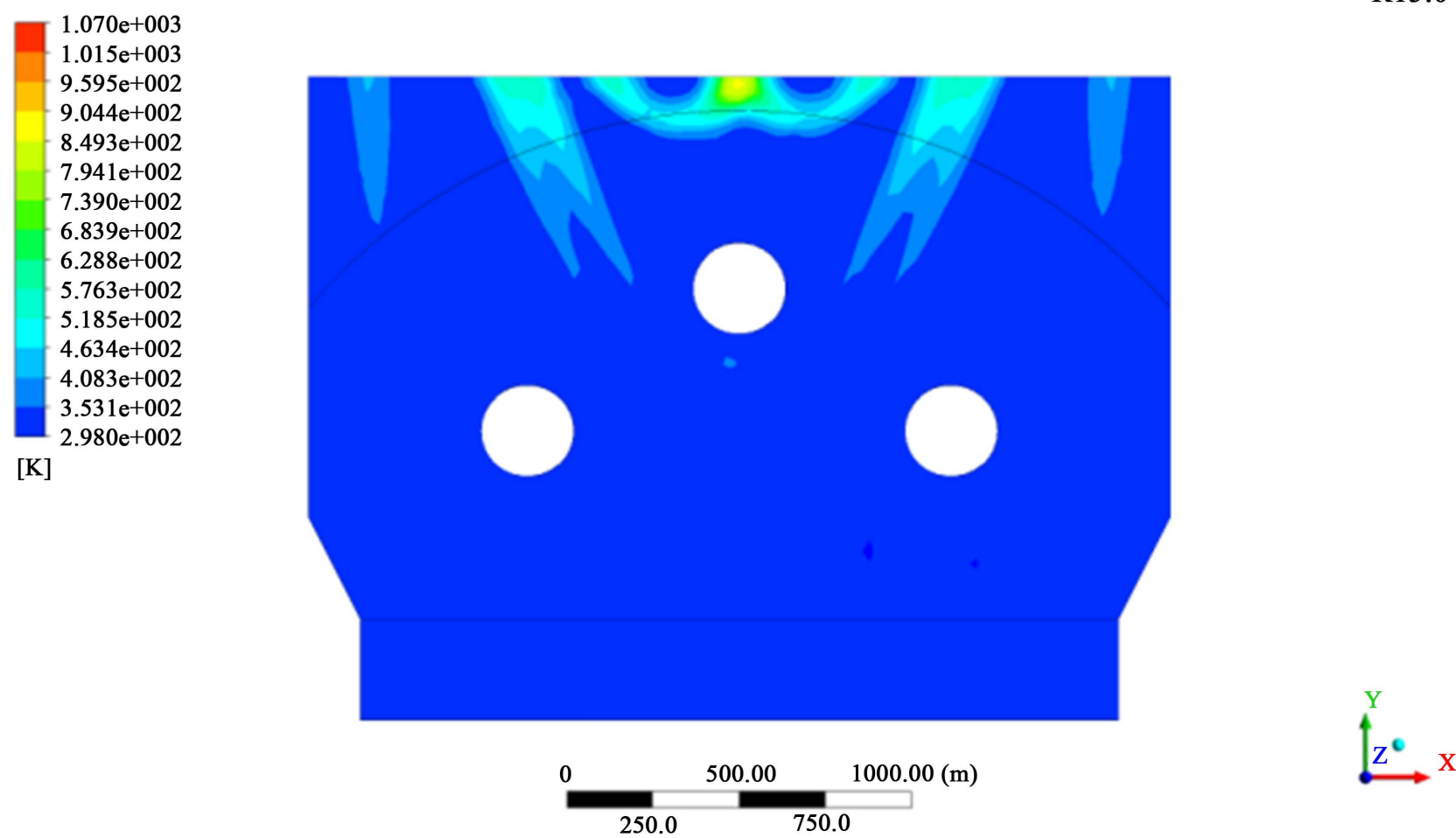

Figure 8. Group 9 structure trolley $1200 \mathrm{~s}$ air temperature distribution cloud.

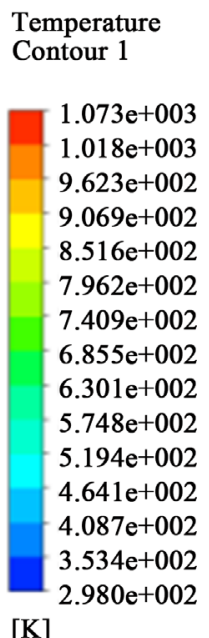

[K]

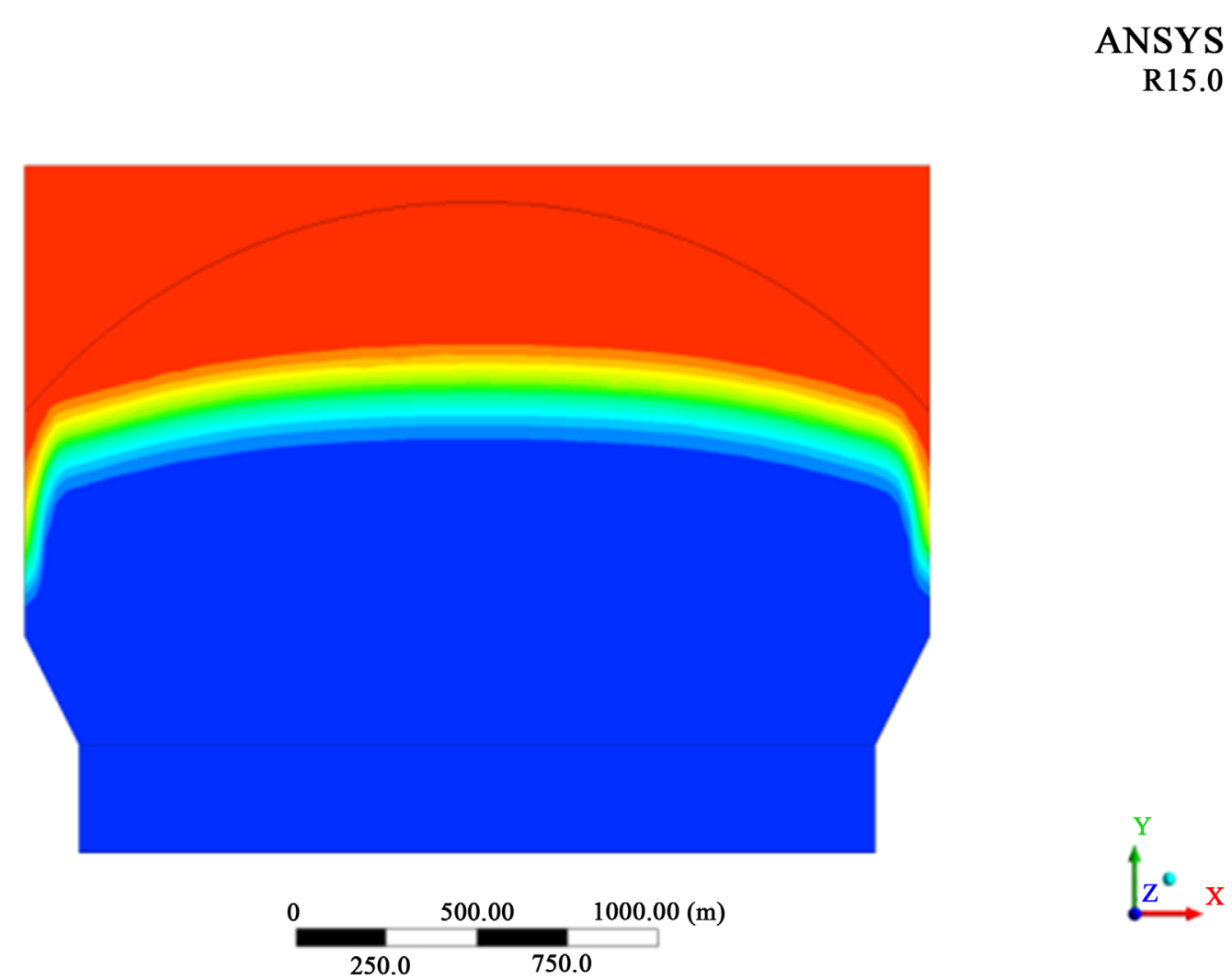

ANSYS

R15.0

$.073 e+003$

$9.623 \mathrm{e}+002$

$9.069 \mathrm{e}+002$

$8.516 \mathrm{e}+002$

$7.962 \mathrm{e}+002$

$6.301 \mathrm{e}+002$

$5.748 \mathrm{e}+002$

$4.641 \mathrm{e}+002$

. $087 \mathrm{e}+002$

$3.534 \mathrm{e}+002$

Figure 9. Traditional structure trolley $1200 \mathrm{~s}$ air temperature distribution cloud. 
Temperature

Contour 1

ANSYS

R15.0

$4.641 \mathrm{e}+003$

$4.522 \mathrm{e}+003$

$4.404 \mathrm{e}+002$

$4.285 \mathrm{e}+002$

$4.166 \mathrm{e}+002$

$4.048 \mathrm{e}+002$

$3.929 \mathrm{e}+002$

$3.811 \mathrm{e}+002$

$3.692 \mathrm{e}+002$

$3.573 \mathrm{e}+002$

$3.455 \mathrm{e}+002$

$3.336 \mathrm{e}+002$

$3.217 \mathrm{e}+002$

$3.099 \mathrm{e}+002$

$2.980 \mathrm{e}+002$

[K]
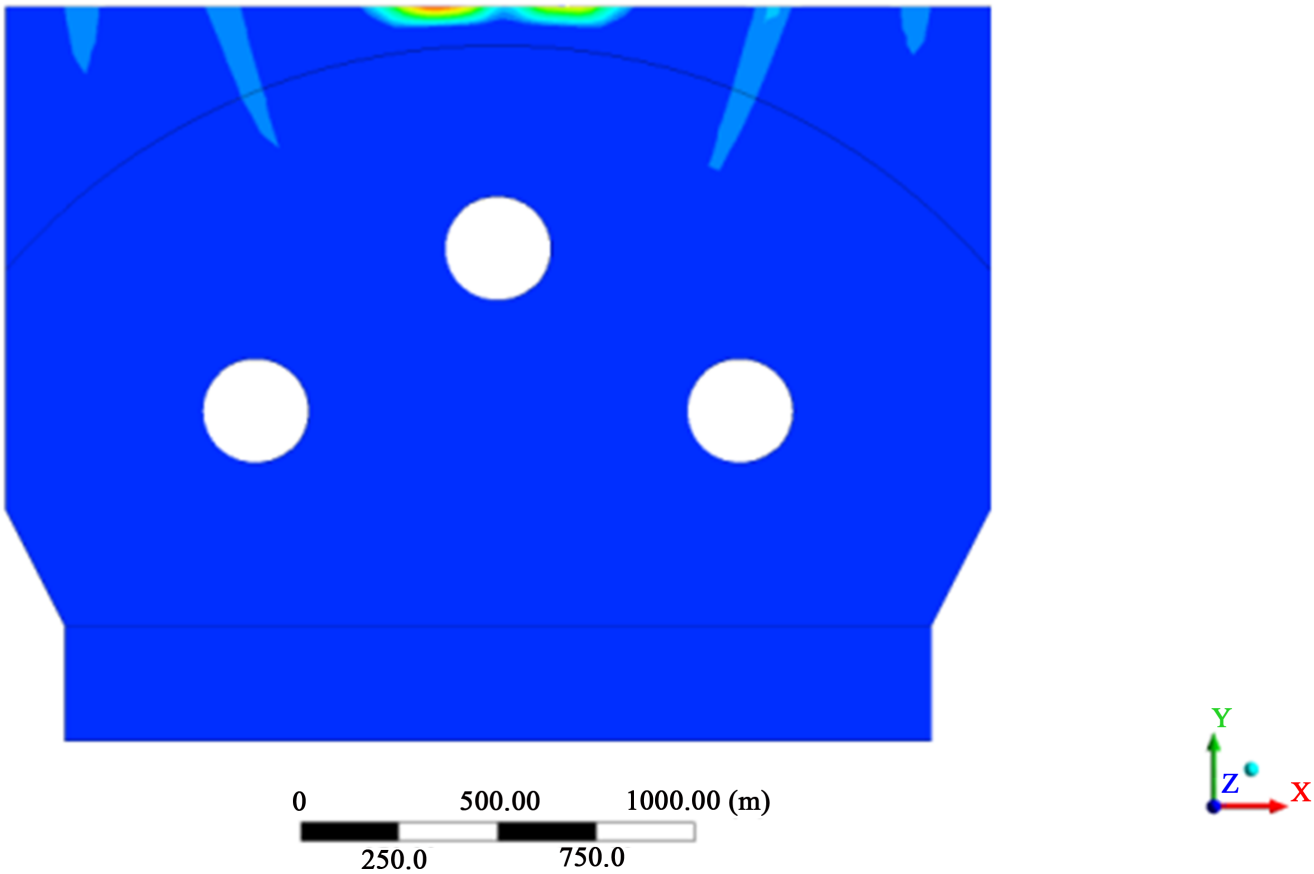

Figure 10. Group 9 structure trolley 1600 s air temperature distribution cloud.

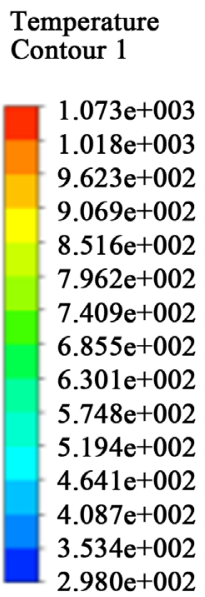

[K]

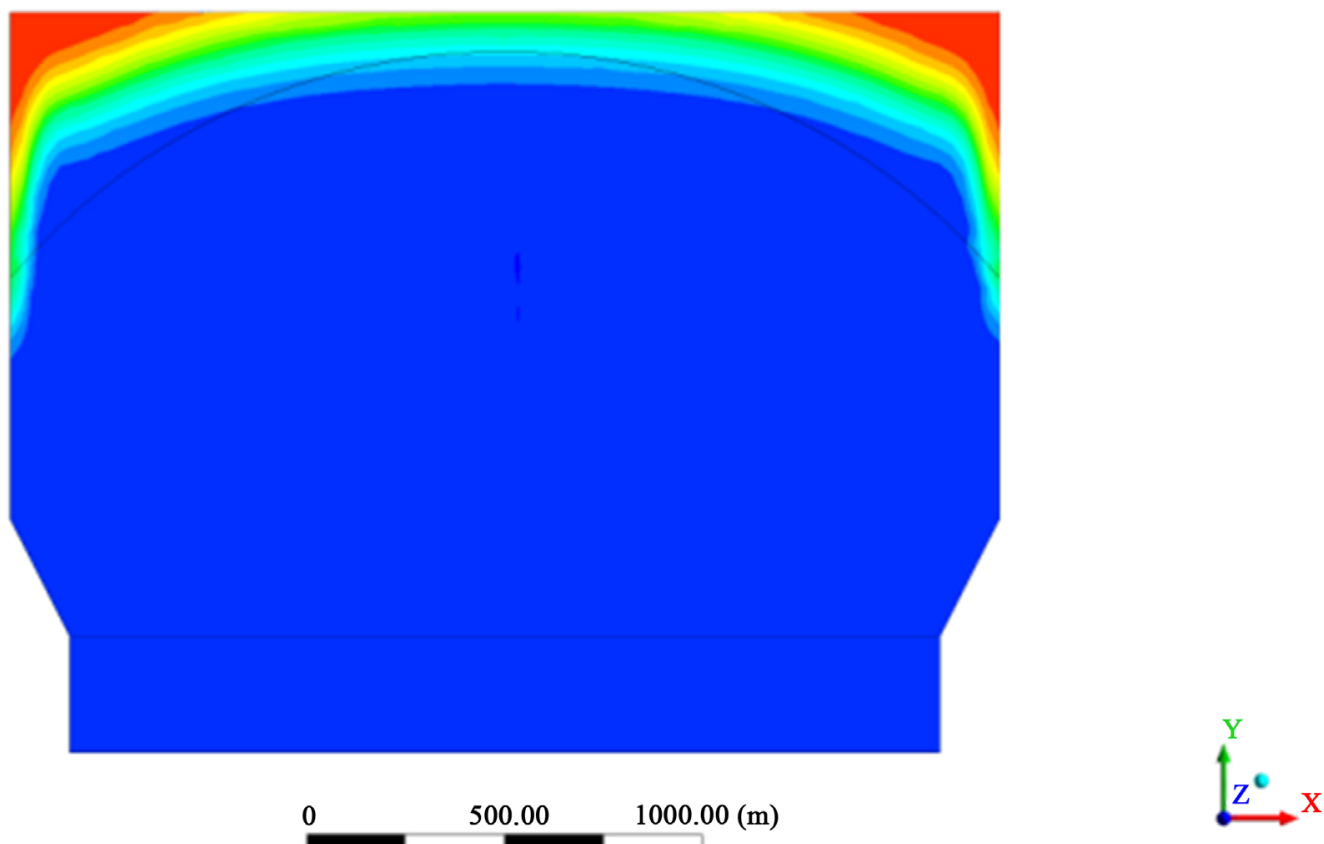

Figure 11. Traditional structure trolley $1600 \mathrm{~s}$ air temperature distribution cloud. 
structure of the porous tube trolley has reduced $41 \%$ cooling time and increased $50 \%$ waste heat recovery

3) In this paper, when the model is numerically simulated, the sintering process is simplified; it only considered the convective heat transfer and ignored other forms of heat transfer, such as the radiation heat transfer of the sinter and the thermal conductivity of the trolley wall. Therefore, follow-up work proposed to take the radiation heat transfer model and wall heat transfer into consideration, to make the numerical simulation more consistent with reality.

\section{Acknowledgements}

The authors would like to acknowledge the Key Subject of Hunan Provincial Department of Education (17A183) support and encouragement to accomplish this work.

\section{References}

[1] Tang, X.J. (2008) China’s Sintering Industry, Technological Progress. Sintering Pellets, 33, 1-4.

[2] Pan, H. (2007) Sintering Circular Cooler Machine System Test Technology and Optimization Research. Liaoning University of Science and Technology.

[3] Feng, J.X., Liang, L.L., Zhang, M., et al. (2010 Mathematical Model of Thermal Process of Pellets in Ring Cooler. Reported, 32, 1596-1600.

[4] Dong, H., Li, J., Luo, Y.Q., et al. Experimental study on the Cooling Process of Sinter. Journal of Northeast University (Natural Science), No. 5, 689-692.

[5] Zhao, B., Zhao, L.J., Qu, T.T., et al. (2013) Relevant Correlation Field of Gas-Solid Heat Transfer Experiment in Sinter Channel of Annular Cooling Machine. Thermal Science and Technology, 12, 302-306.

[6] Zhang, X.H., Zhang, J.Y., Dai, C.D., et al. (2011) Numerical Simulation and Optimization of the Cooling Process of Sinter. Acta Chimica Sinica, 62, 3081-3087.

[7] Wang, F.J. (2013) Computational Fluid Dynamics Analysis. Tsinghua University Press, Beijing, 11.

[8] Shang, X.D. (2013) Optimization of Multiphase Flow of Blast Furnace Based on Computational Fluid Dynamics. Beijing University of Chemical Technology.

[9] Fluent User's Guide. Fluent Inc. 14.5.

[10] Zhang, J.Y., Tian, W.Y., Dai, C.D., et al. (2012) Simulation and Optimization of Stratified Fabric of Circular Cooler. Acta Chimica Sinica, 63, 1385-1390.

[11] Zhao, H.G., Hu, S.Z. and Mao, Y.Z. (2009) Lean Iron Sintering Machine Exhaust Waste Heat Recovery Practice. Shandong Metallurgy, 31, 12-14.

[12] Zhu, M.K., Qing, D.F., Liu, S.X., et al. (2016) Research on Built-In Horizontal Pipe Structure and numerical Simulation of Annular Cooling Machine. Journal of South of China University (Natural Science Edition), 30, 22-27. 